\title{
Stress reactivity and emotion in premenstrual syndrome
}

\author{
This article was published in the following Dove Press journal: \\ Neuropsychiatric Disease and Treatment \\ 16 June 2017 \\ Number of times this article has been viewed
}

\author{
Qing Liu' \\ Yongshun Wang ${ }^{2}$ \\ Cornelis Hermanus van \\ $\mathrm{Heck}^{3}$ \\ Wei Qiao ${ }^{4}$ \\ 'Department of Nuclear Medicine \\ and Medical PET Center, The Second \\ Hospital of Zhejiang University \\ School of Medicine, Zhejiang \\ University, Hangzhou, ${ }^{2}$ School of \\ Physical Education and Sport, Huaqiao \\ University, Xiamen, People's Republic \\ of China; ${ }^{3} \mathrm{DCC}$, Donders Institute for \\ Neuroscience and Neurocognition, \\ Arnhem, the Netherlands; \\ ${ }^{4}$ Department of Physical Education, \\ Xiamen Institute of Technology, \\ Xiamen, People's Republic of China
}

Correspondence: Wei Qiao

Department of Physical Education,

Xiamen Institute of Technology,

I25I sunbannan Road, Jimei District,

Xiamen, People's Republic of China

$\mathrm{Tel} / \mathrm{fax}+865926162116$

Email lemonpsy@outlook.com
Background: Hormone level fluctuation across the menstrual cycle causes women to experience negative emotions and also affects their mood regulation and stress sensitivity. However, the stress reactivity and emotional variations in women with premenstrual syndrome (PMS), who are especially sensitive to the variations in hormone cycles, have not been explained.

Methods: The present study used an electroencephalogram (EEG) stress evaluation test, a physiology stress evaluation test, and the positive affect and negative affect scale (PANAS) to evaluate the stress reactivity pattern and emotional state of women with PMS.

Results: The results showed that women with PMS had higher negative affect and lower positive affect compared with controls. Moreover, under stressful conditions, the women with PMS had a higher alpha activity and a lower respiration rate than the controls. The differences in stress reactivity and emotional states between women with PMS and controls were based on a covariant analysis with menstrual cycle (luteal and follicular phases) as the covariate.

Conclusion: The results demonstrated that, compared with controls, women suffering from PMS have a continuous abnormality in emotional state and stress reactivity, which was independent of the menstrual cycle.

Keywords: premenstrual syndrome, stress reactivity, emotion, EEG stress evaluation test, physiology stress evaluation test

\section{Introduction}

Most women of reproductive age may feel more physiological and/or emotional discomfort in the week before the menses. These symptoms can vary between individuals and have the potential to affect work, personal life, and place additional stress on a relationship. ${ }^{1,2}$ In all, 30\%-40\% of women of reproductive age suffer from the more severe premenstrual syndrome (PMS) and 3\%-8\% suffer from premenstrual dysphoric disorder (PMDD), which is the more serious variant of PMS. ${ }^{3}$ The symptoms of PMS cover emotion, physiology, and behavioral fields and can be related to the menstrual cycle. These symptoms typically occur in the premenstrual or luteal phase of the menstrual cycle and tend to vanish in or near the end of the cycle. ${ }^{4}$ The exact etiology of PMS is unknown, but it may be related to hormone variations. ${ }^{5}$ Some theories state that PMS is not caused by abnormal concentration of gonadal steroids but more likely by variations in levels of the sex hormones. The differences between women with and without PMS may also be explained by increased sensitivity to variations in levels of sex hormones. ${ }^{6}$ Moreover, some studies showed that the onset and course of PMS are related to stress. ${ }^{7}$

The variations in hormone levels across the menstrual cycle cause an increase in negative emotions in women and can influence mood regulation and sensitivity to stress..$^{8,9}$ Specifically, women have stronger responses to stressors before the menstruation or 
in the luteal phase, which may increase the risk for negative emotions or moods, suggesting stress may strengthen PMS symptoms. ${ }^{10}$ Lustyk et $\mathrm{al}^{11}$ made a distinction between a "high symptom" group and a "low symptom" group in women with PMS (N=114) on the severity of PMS symptoms. Their results showed that, compared with low symptom group, the high symptom group reported more stressful experiences. Previous studies have also found that stressful experiences of women with PMDD before the menses were mainly related to a higher stress sensitivity. ${ }^{12,13}$

Although the abovementioned studies investigated the relationship of menstrual cycle, PMS, and stress, the results are inconclusive. A major issue was the lack of a comparison between the stress reactivity of women suffering from PMS and healthy controls, in confronting the same physiological and psychological stressors. In addition, the measurements of stress reactivity in the foregoing studies were based on the subjective reports by the participants themselves, which lack objective neural and physiological indexes.

Therefore, the present study adopted an electroencephalogram (EEG) stress evaluation test, a physiology stress evaluation test, and the positive affect and negative affect scale (PANAS) to investigate the stress reactivity pattern and emotional state of women with PMS and healthy controls. Based on the previous research, ${ }^{2}$ we hypothesize that women with PMS may have more subjective concerns as well as abnormal neural activity and physiological reactivity under stressful conditions, when compared with healthy controls.

\section{Methods}

\section{Ethics}

All participants provided written informed consent before participating in the study. Experimental procedures were approved by the institutional review board of the State Key Laboratory of Cognitive Neurosciences and Learning of Beijing Normal University.

\section{Participants}

Before the experiment, a gynecological examination and B-mode ultrasonic echo were conducted on participants to eliminate other disorders and disease, as well as confirm the absence of past surgery. As such, it was confirmed that the participants were healthy. We also used PMS scales ${ }^{14}$ and self-compiled historic information (may contain retrospective recall biases) on the women's menstrual cycles to screen and group the 86 participants. The participants were recruited via flyers in the university and the campus network. Exclusion criteria consisted of being currently pregnant or lactating,
Table I Sociodemographic characteristics (mean \pm SD) of both groups (PMS and control groups)

\begin{tabular}{lll}
\hline & PMS & Control \\
\hline $\mathrm{N}$ & 15 & 15 \\
Age (years) & $23 \pm 1$ & $22 \pm 2$ \\
Menophania (years) & $13 \pm 1$ & $13 \pm 1$ \\
The phase of MC in pretest & 9 in LP, 6 in FP & 6 in LP, 9 in FP \\
The phase of MC in posttest & 7 in LP, 8 in FP & 7 in LP, 8 in FP \\
Cycle length (days) & $29 \pm 2$ & $28 \pm 3$ \\
\hline
\end{tabular}

Note: LP: I-3 days before the menstruation and FP: I-3 days after the menstruation. Abbreviations: PMS, premenstrual syndrome; MC, menstrual cycle; LP, luteal phase; FP, follicular phase.

taking oral contraceptives, receiving medical treatment of any kind, taking medicine that could affect stress reactivity, having a personality disorder, clinical anxiety, depression, and abnormal psychological syndrome, or having irregular menstrual cycles.

Prospective self-reports about the start of the menses were combined with primary gynecological examination and B-ultrasonic wave results, to schedule the experiment. According to the individual physical character of the participants' menstrual cycle and the hormone levels, all participants (both the PMS and control group) were tested twice; once in the luteal phase (1-3 days before the menstruation) and once in the follicular phase (1-3 days after the menstruation). The final sample was composed of 30 participants (22 \pm 2.19 years old), of whom 15 females were in the PMS group and 15 females were in the healthy control group. The demographic information of participants is shown in Table 1.

\section{Materials}

\section{PMS scale}

The PMS scale consisted of 12 items that are related to emotional and physical symptoms and gave the degree of severity of PMS. ${ }^{14}$ The items were scored on a four-point scale, with 0 as "no symptoms" and 3 as "symptoms seriously affecting life, study and work, needing treatment". Total scores $>6$ indicated the presence of PMS. The Chinese version of the PMS scale had good reliability and validity. ${ }^{15}$

\section{PANAS}

The PANAS includes 20 items that are divided equally into positive and negative affect dimensions. Participants indicate how much a statement applies to them using a 5-point Likert scale, where 1 represents "none" and 5 "very much". ${ }^{16}$ Summing the items in the two categories generates the "positive affect score" and the "negative affect score". As such, 
the scores of positive affect and negative affect range from 10 to 50 . Both the validity and reliability of the PANAS are well established and considered to be effective tools for the evaluation of emotional state. ${ }^{17}$

\section{Electroencephalogram and physiological stress evaluation tests}

An Infiniti3000A (ProComp Infiniti; Thought Technology, Montreal, Canada) biofeedback system (including computer, Bioneuro Infiniti encoder, EEG electrodes, and physiological sensors) was used to conduct the electroencephalogram stress evaluation test and the physiology stress evaluation test.

The Infiniti3000A was used with a unipolar EEG electrode to conduct the EEG stress evaluation test, which uses three leads: one disk electrode, one ear clip reference electrode, and one ear clip ground electrode. The disk electrode was set at $\mathrm{Cz}$, according to the international 10-20 electrode system. The " 10 " and " 20 " refer to the fact that the actual distances between adjacent electrodes are either $10 \%$ or $20 \%$ of the total front-back or right-left distance of the skull. The EEG stress evaluation tests included a baseline test, an attention test, and a cognition test. The EEG sensor recorded the analog EEG signal and converted it to a digital signal for analysis. The main EEG parameter used was the alpha wave amplitude (8-13 Hz, amplitude 20-100 $\mu \mathrm{V})$, which is related to the relaxed mental state.

Physiological stress was recorded through the Infiniti3000A as well, using electromyography (EMG), skin conductance (SC), heart rate (HR), and respiration rate to estimate physiological reactivity. EMG was recorded using the MyoScan-Pro sensor, which is a head-worn unipolarelectrode (including positive, negative, and reference leads) mounted on the middle of the frontal region, on the occipitofrontal muscle. EMG amplitude below $5 \mu \mathrm{v}$ was interpreted as a relaxed state, while an amplitude between 5 and $15 \mu \mathrm{v}$ was interpreted as mild tenseness. EMG amplitude above $15 \mu \mathrm{v}$ was interpreted as high tenseness. The SC sensor electrode was mounted on the tip of the ring finger of the dominant hand. The value of SC below 5 mho was taken to correspond with a relaxed state, while a value between 5 and 10 mho was taken to correspond with mild tenseness. A value between 10 and 20 mho was taken to correspond with tenseness, while a value above 20 mho was taken to correspond with anxiety and high tenseness. HR was recorded using a blood volume pulse sensor (BVP), which was mounted on the tip of the middle finger of the dominant hand. The BVP measures the blood flow through changes in infrared reflection and represents a reliable and stable measure of real-time HR.
The HR is $75 \mathrm{bpm}$ for normal adults in a state of relaxation. The respiration sensor (Resp) was placed on the participants' abdomen.

The physiological stress evaluation test battery consisted of a baseline measurement, the color-word test, the first rest interval, the math test, the second rest interval, the stress event recall test, third, and final rest intervals. We computed the baseline test value and the values of the rest intervals for the evaluation of physiological stress reactivity. The baseline test value represents the baseline of stress reactivity, which belongs to the first stage of stress reactivity. The values of the first rest interval represent the effects of the color-word test on the physiology. The values of the second rest interval represent the effects of the math test. By combining, these values belong to the second stage of stress reactivity. The values of the third rest interval represent the effect of the stress event recall test on the physiology and were also used as the index of stress resilience, which is the third stage of stress reactivity.

\section{Procedure}

To provide a comfortable and stable testing environment, the testing room was kept clean, well ventilated, and dimly lit. Before the experiment, the participants filled in informed consent forms, as well as the PANAS. Before the connection of biofeedback equipment, the participants were presented with the operating principle of the biofeedback machine and the used methods. After the connection of the equipment, the participants were asked to sit in a comfortable manner and relax. The EEG stress evaluation test included three stages. The first stage was 2 minutes of baseline resting EEG. The second stage was a 3-minute attention test, and the third stage was a 3-minute cognition test. There were instructions between stages to guide the participants into the next stage. The EEG was recorded for each stage. The total time of the EEG stress evaluation test was 15 to 20 minutes. Next, the physiological stress evaluation test was carried out, which included seven stages. The first stage was the baseline stage, which lasted for 2 minutes. The second stage was the colorword test, which lasted for 3 minutes. The third stage was the first rest interval, which lasted for 2 minutes. The fourth stage was the math test, which lasted for 3 minutes. The fifth stage was the second resting interval, which lasted for 2 minutes. The sixth stage was the stress recall test which lasted for 3 minutes, and the last stage was the third resting interval, which lasted for 2 minutes. The total time of the physiology stress evaluation test was 20 to 30 minutes. The specific procedure is shown in Figure 1. 


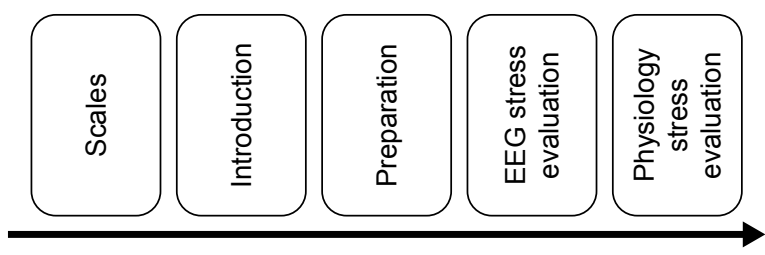

Figure I Timeline of the experimental condition. Abbreviation: EEG, electroencephalogram.

\section{Data analysis}

All the statistics were conducted using SPSS 16.0 software. Specifically, mixed-factor analyses of variance (ANOVAs) were performed on the EEG alpha activity, and physiology data of the physiological stress evaluation test. The within-subject variable was the TEST ("resting, attention, and cognition tests" for EEG alpha activity; "baseline, rest 1 , rest 2 , and rest tests for physiology data"), whereas the between-subject variable was GROUP (PMS vs control group). The PHASE (luteal and follicular phases) was the covariate.

\section{Results}

\section{The positive affect and negative affect of PMS and control groups}

Independent sample $t$-tests were conducted for the PANAS scores for women with PMS and healthy control separately. The results showed that there were significant differences in the positive affect $\left(t_{28}=-1.782, P=0.086, d=-0.65\right)$ and negative affect $\left(t_{28}=1.852, P=0.080, d=0.68\right)$ between these two groups. Compared with the healthy controls the women with PMS had a lower score for positive affect (Figure 2).

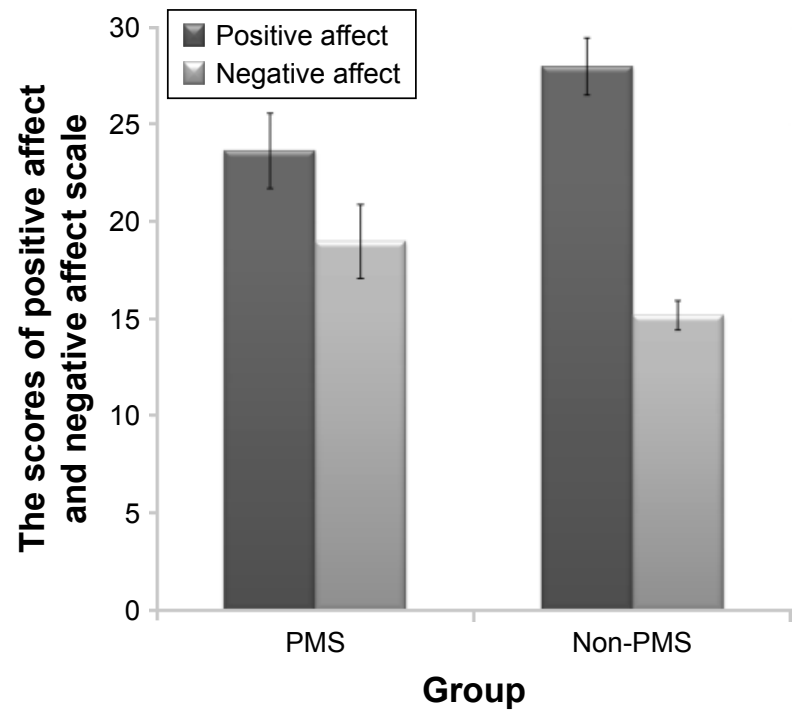

Figure 2 The scores of positive affect and negative affect for PMS group ( $N=15)$ and control group ( $\mathrm{N}=15)$.

Abbreviation: PMS, premenstrual syndrome.

\section{The EEG stress evaluation test results of} PMS and control groups

The results indicated that the main effect of GROUP $\left(F_{(1,27)}=18.765, P<0.001, \eta^{2}=0.410\right)$ on alpha activity was significant. The main effect of TEST $\left(F_{(2,54)}=1.138, P=0.328\right.$, $\left.\eta^{2}=0.040\right)$ on alpha activity was not significant. However, the interaction of GROUP and TEST on alpha activity was significant, $F_{(2,54)}=3.562, P=0.035, \eta^{2}=0.117$. Post hoc analysis of the significant interaction revealed that the alpha activity of PMS group under rest $\left(F_{(1,28)}=25.85, P<0.001\right)$, attention test $\left(F_{(1,28)}=14.10, P=0.001\right)$, and cognition test $\left(F_{(1,28)}=10.06\right.$, $P=0.04$ ) was higher than the control group (Figure 3).

\section{The physiological stress evaluation test results of PMS and control groups}

The results showed that the main effects of GROUP on EMG, HR, and SC were not significant $\left(F_{E M G(1,27)}=0.098\right.$, $p_{E M G}=0.756, \eta_{E M G}^{2}=0.004 ; F_{B V P(1,27)}=1.991, p_{B V P}=0.170$, $\left.\eta_{B V P}^{2}=0.069 ; F_{S C(1,27)}=0.026, p_{S C}=0.872, \eta_{S C}^{2}=0.001\right)$. The main effects of TEST on EMG, HR, and SC were also not significant $\left(F_{E M G(3,81)}=1.631, p_{E M G}=0.189, \eta_{E M G}^{2}=0.057\right.$; $F_{B V P(3,81)}=1.488, p_{B V P}=0.224, \eta_{B V P}^{2}=0.052 ; F_{S C(3,81)}=1.918$, $\left.p_{S C}=0.133, \eta_{S C}^{2}=0.066\right)$. And the interaction of GROUP and TEST on EMG, HR, and SC was not significant either $\left(F_{E M G(3,81)}=1.428, p_{E M G}=0.241, \eta_{E M G}^{2}=0.050 ; F_{B V P(3,81)}=0.564\right.$, $p_{B V P}=0.640, \eta_{B V P}^{2}=0.020 ; F_{S C(3,81)}=0.488, p_{S C}=0.692$, $\left.\eta_{S C}^{2}=0.018\right)$.

However, the main effect of GROUP on respiration rate was significant, $F_{(1,27)}=4.132, P=0.052, \eta^{2}=0.133$. The main effect of TEST on respiration rate was significant, $F_{(3,81)}=8.681, P<0.001, \eta^{2}=0.243$, and the interaction of

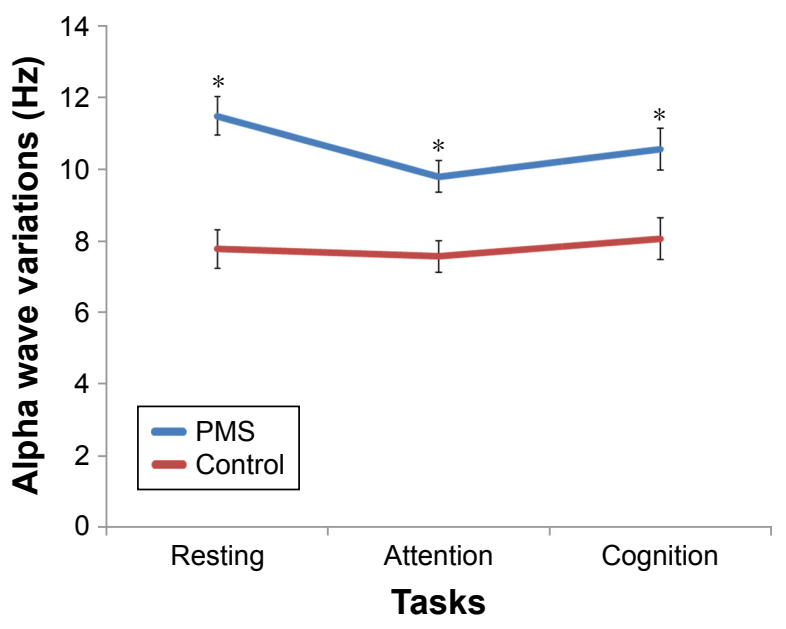

Figure 3 The EEG alpha wave $(\mathrm{Hz})$ under different tasks (resting, attention, and cognition tasks) of EEG stress evaluation test for PMS group $(\mathrm{N}=15)$ and control group $(\mathrm{N}=15)$. $* \mathrm{P}<0.05$.

Abbreviations: EEG, electroencephalogram; PMS, premenstrual syndrome. 


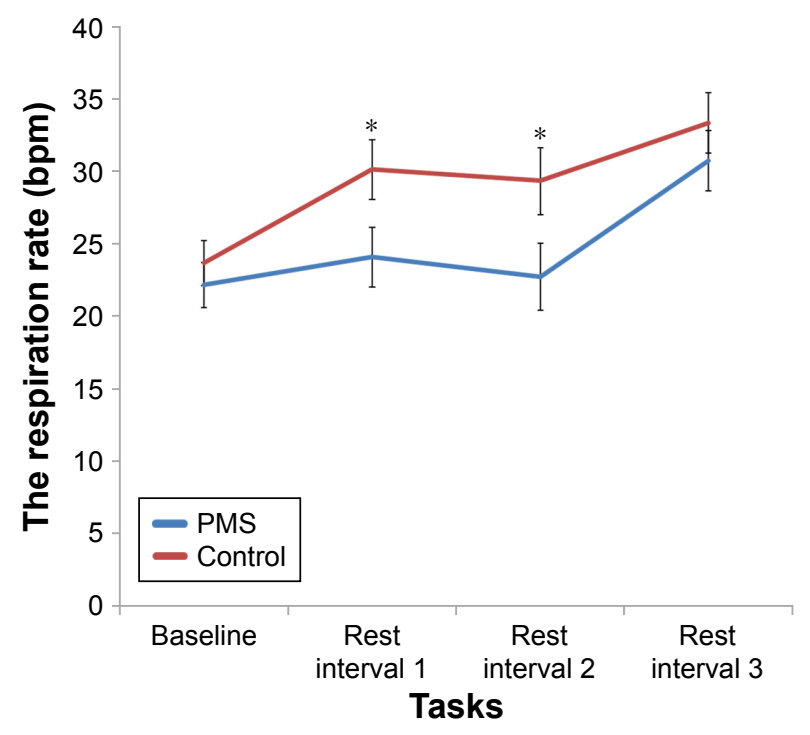

Figure 4 The respiration rate (bpm) under different tasks (baseline, rest interval I, rest interval 2, and rest interval 3 tasks) of physiology stress evaluation tests for PMS group $(\mathrm{N}=15)$ and control group $(\mathrm{N}=\mid 5)$. $* P<0.05$.

Abbreviation: PMS, premenstrual syndrome.

GROUP and TEST was also significant, $F_{(3,81)}=3.224$, $P=0.027, \eta^{2}=0.107$. Post hoc analysis found that the respiration rates of PMS group during rest interval $1\left(F_{(1,28)}=4.06\right.$, $P=0.054)$ and rest interval $2\left(F_{(1,28)}=4.29, P=0.049\right)$ were significantly lower than the control group (Figure 4).

\section{Discussion}

The present study investigated the differences in neurophysiological reactivity and emotional state to stressors in women with PMS compared with healthy controls. As expected, we found that compared with healthy controls, the women with PMS had higher negative affect and lower positive affect. In addition, under stressful conditions, compared with healthy controls, women with PMS had a more pronounced EEG alpha activity and lower respiration rates. The menstrual cycle (luteal and follicular phases) was used as the covariate in the analyses. In other words, compared with healthy females, women suffering from PMS had a continuous abnormality in emotional state and stress reactivity, which was independent of the menstrual cycle. Specifically, the women had a lower positive affect and a higher negative affect, together with stronger alpha wave activity, as well as an inhibition of the physiological response (lower respiration rate) when confronted with stressors.

Ossewaarde et $\mathrm{al}^{18}$ investigated the neural mechanism of stress sensitivity interactions with the menstrual cycle and found that the natural fluctuation of allopregnanolone may influence females' stress sensitivity. Since women with PMS are more sensitive to allopregnanolone fluctuations, they would show increased stress sensitivity and reactivity when compared with healthy controls. This may explain the more pronounced EEG alpha activity, which we observed in women with PMS. The relationship between sensitivity to sex hormones and the stress reactivity of women with PMS is also supported at the neural level. The fact that function and even the anatomy of the amygdala are regulated by sex hormones has been extensively proven., ${ }^{8,19,20}$ The amygdala is involved with detection of potential threats and responses to anger and fear stimuli and is activated in "fight or flight" behavior, as well as involved in the stress reactivity system. ${ }^{21}$ As the amygdala has been implicated in the generation or maintenance of activity in the alpha spectrum, and in this study, we show increased alpha activity as well, it would follow that the amygdala is involved in the generation or maintenance of PMS.

We observed that, compared with healthy controls, women with PMS have lower respiration rates after the color-word and math tests. This showed that women with PMS were more physiologically affected by the stress tests. Women with PMS lack the flexibility to stimulate their physiological resources, which results in an increase in respiratory rate to stressors when compared with healthy controls. So, the lower respiration rate of women with PMS can reflect more "inhibition" of physiological stressors. We did not get the fast rise of HR in PMS groups, as in a study using the Trier Social Stress Test (TSST), ${ }^{22}$ because this needs more involvement of the participants and social interaction. ${ }^{23}$ In contrast, our stress test involved mainly cognitive loads. Moreover, women with PMS have a distinctly different stress reactivity pattern compared with the control group, which means their stress coping mechanism is possibly incomparable.

The present study demonstrated that, compared with healthy controls, women with PMS experience more negative affect and less positive affect and also show a more pronounced alpha activity as well as lower respiration rates after being exposed to stressful conditions. These effects were independent of the menstrual cycle, which meant that continuous and permanent changes have occurred in women with PMS. However, the study may be underpowered to assess significant differences due to low sample size.

\section{Acknowledgments}

The work was funded by Huaqiao University's Academic Project, which was supported by the Fundamental Research Funds for the Central Universities (15SKGC-QG15). The authors would like to express their gratitude for the support of these projects. 


\section{Disclosure}

The authors report no conflicts of interest in this work.

\section{References}

1. Sigmon ST, Dorhofer DM, Rohan KJ, Boulard NE. The impact of anxiety sensitivity, bodily expectations, and cultural beliefs on menstrual symptom reporting: a test of the menstrual reactivity hypothesis. $J$ Anxiety Disord. 2000;14(6):615-633.

2. Sigmon ST, Schartel JG, Herman BA, Cassel AG, Thorpe GL. The relationship between premenstrual distress and anxiety sensitivity: the mediating role of rumination. J Rat Emo Cognitive Behav Ther. 2009; 27:188-200.

3. Ryu A, Kim TH. Premenstrual syndrome: a mini review. Maturitas. 2015;82(4):436-440.

4. Indusekhar R, Usman SB, O’Brien S. Psychological aspects of premenstrual syndrome. Best Pract Res Clin Obstet Gynaecol. 2007; 21(2):207-220.

5. Cubeddu A, Bucci F, Giannini A, et al. Brain-derived neurotrophic factor plasma variation during the different phases of the menstrual cycle in women with premenstrual syndrome. Psychoneuroendocrinology. 2011;36(4):523-530.

6. Yonkers KA, O’Brien PMS, Eriksson E. Premenstrual syndrome. Lancet. 2008;371(9619):1200-1210.

7. Perkonigg A, Yonkers KA, Pfister H, Lieb R, Wittchen HU. Risk factors for premenstrual dysphoric disorder in a community sample of young women: the role of traumatic events and posttraumatic stress disorder. J Clin Psychiatry. 2004;65(10):1314-1322.

8. Ossewaarde L, van Wingen GA, Ripkema M, et al. Menstrual cyclerelated changes in amygdale morphology are associated with changes in stress sensitivity. Hum Brain Mapp. 2013;34(5):1187-1193.

9. Olson KC, Carroll HA, Lustyk MK. Psychophysiological stress reactivity relationships across the menstrual cycle. J Horm. 2015;2015:1-5.

10. Kischabaum C, Kudielka BM, Gaab J, Schommer NC, Hellhammer DH. Impact of gender, menstrual cycle phase, and oral contraceptives on the activity of the hypothalamus-pituitary-adrenal axis. Psychosom Med. 1999; 61(2):154-162.

11. Lustyk MKB, Widman L, Paschane A, Ecker E. Stress, quality of life and physical activity in women with varying degrees of premenstrual symptomatology. Women Health. 2004;39(3):35-44.
12. Epperson CN, Pittman B, Czarkowski KA, Stiklus S, Krystal JH, Grillon C. Luteal-phase accentuation of acoustic startle response in women with premenstrual dysphoric disorder. Neuropsyhophamacology. 2007;32(10):2190-2198.

13. Kask K, Backstrom T, Lundgren P, Poromaa IS. Allopregnanolone has no effect on startle response and prepulse inhibition of startle response in patients with premenstrual dysphoric disorder or healthy controls. Pharmacol Biochem Behav. 2009;92(4):608-613.

14. Bancroft J. The premenstrual syndrome - a reappraisal of the concept and the evidence. Psychol Med. 1993;Suppl 24:1-47.

15. Yu C. Relationship between Premenstrual Syndrome and Type D Personality in Female College Students [Master's thesis]. Central South University, Changsha; 2008:1-38.

16. Tran V. Positive affect negative affect scale (PANAS). Encyclopedia of Behavioral Medicine. 2013;1508-1509.

17. Huang L, Yang T, Ji Z. Applicability of the positive and negative affect scale in Chinese. Chin Ment Health J. 2003;17:54-56.

18. Ossewaarde L, Hermans EJ, van Wingen GA, et al. Neural mechanisms underlying changes in stress-sensitivity across the menstrual cycle. Psychoneuroendocrinology. 2010;35(1):47-55.

19. Andreano JM, Cahill L. Menstrual cycle modulation of medial temporal activity evoked by negative emotion. Neuroimage. 2010;53(4): 1286-1293.

20. Choi JC, Park SK, Kim YH, et al. Different brain activation patterns to pain and pain-related unpleasantness during the menstrual cycle. Anesthesiology. 2006;105(1):120-127.

21. Van Wingen GA, Ossewaarde L, Backstrom T, Hermans EJ, Fernandez G. Gonadal hormone regulation of the emotion circuitry in humans. Neuroscience. 2011;191:38-45.

22. Dickerson SS, Kemeny ME. Acute stressors and cortisol responses: a theoretical integration and synthesis of laboratory research. Psychol Bull. 2004;130(3):355-391.

23. Villada C, Espin L, Hidalgo V, Rubagotti S, Sgoifo A, Salvador A. The influence of coping strategies and behavior on the physiological response to social stress in women: the role of age and menstrual cycle phase. Physiol Behav. 2017;170:37-46.
Neuropsychiatric Disease and Treatment

\section{Publish your work in this journal}

Neuropsychiatric Disease and Treatment is an international, peerreviewed journal of clinical therapeutics and pharmacology focusing on concise rapid reporting of clinical or pre-clinical studies on a range of neuropsychiatric and neurological disorders. This journal is indexed on PubMed Central, the 'PsycINFO' database and CAS,

\section{Dovepress}

and is the official journal of The International Neuropsychiatric Association (INA). The manuscript management system is completely online and includes a very quick and fair peer-review system, which is all easy to use. Visit http://www.dovepress.com/testimonials.php to read real quotes from published authors. 